\title{
Phosphodiesterase-4 Inhibition in Psoriasis
}

This article was published in the following Dove Press journal:

Psoriasis: Targets and Therapy

\author{
Milica Milakovic (iD) \\ Melinda J Gooderham $\mathbb{1 D}^{2-4}$ \\ 'Faculty of Medicine, University of \\ Toronto, Toronto, ON, Canada; ${ }^{2}$ Skin \\ Centre for Dermatology, Peterborough, \\ ON, K9J 5K2, Canada; ${ }^{3}$ Probity Medical \\ Research, Waterloo, ON, N2J IC4, \\ Canada; ${ }^{4}$ Department of Medicine, \\ Queen's University, Kingston, ON, K7L \\ 3N6, Canada
}

\begin{abstract}
Psoriasis is a chronic immune-mediated inflammatory disorder. Phosphodiesterase4 (PDE-4) is an enzyme that mediates inflammatory responses and plays a role in psoriasis pathogenesis. PDE-4 degrades its substrate cyclic adenosine monophosphate (cAMP) to adenosine monophosphate (AMP), which subsequently leads to the production of proinflammatory mediators. Inhibitors of PDE-4 work by blocking the degradation of cAMP, which leads to a reduction in inflammation. Apremilast is the only approved oral PDE-4 inhibitor for the treatment of psoriasis. While it is effective for some patients, it may be limited by adverse effects in others. A topical PDE-4 inhibitor, roflumilast, is being investigated in psoriasis and showing promising results. Crisaborole, a topical PDE-4 inhibitor approved for use in atopic dermatitis, has also been investigated in psoriasis. This is an updated comprehensive review to summarize the currently available evidence for the PDE- 4 inhibitors apremilast, roflumilast and crisaborole in the treatment of psoriasis, with a focus on data from randomized clinical trials.
\end{abstract}

Keywords: psoriasis, PDE-4, phosphodiesterase inhibitors, topical therapies

\section{Introduction}

Psoriasis is a common, chronic, immune-mediated inflammatory disorder characterized by erythematous plaques with silvery scale, numerous comorbidities and a significant impact on the quality of life. ${ }^{1}$ Psoriasis immunopathogenesis involves multiple inflammatory mediators, including tumor necrosis factor (TNF)- $\alpha$, interleukin (IL)-12, IL-17, IL-22, IL-23 and interferon (IFN)- $\gamma .^{2}$ Various treatment modalities for psoriasis exist ranging from topical corticosteroids, retinoids and calcipotriol, to oral conventional systemic treatments which act as broad immunosuppressants, to biological therapies which target specific cytokines or receptors to control downstream inflammation. Treatment choices are often based on the severity of disease with mild disease often managed with topical therapy, and moderate-to-severe disease requiring systemic therapy for control. Though topical corticosteroids (TCS) are commonly used and are often effective at treating psoriatic plaques, their long-term side effects, such as atrophy and striae limit their usage. $^{3}$ This is particularly true in more sensitive areas, such as the face or intertriginous areas. Irrespective of the treatment chosen, the same priorities of psoriasis management remain: to provide a highly efficacious treatment that targets a spectrum of inflammatory mediators involved in the pathogenesis of psoriasis, while ensuring a favourable safety profile and limited adverse effects. ${ }^{2}$

Inhibition of the cyclic adenosine monophosphate (cAMP) pathway was first proposed over two decades ago for the management of psoriasis and remains a promising treatment target today. ${ }^{2}$ cAMP is a key secondary messenger that is
Correspondence: Melinda J Gooderham Skin Centre for Dermatology, 775 Monaghan Road, Peterborough, ON, K9] $5 \mathrm{~K} 2$, Canada

Tel + I 7057757546

Fax + I 7057753376

Email mgooderham@centrefordermatology. com
Psoriasis: Targets and Therapy 2021:1। 21-29

21 
expressed in all cells and is intimately involved in a wide variety of normal cellular responses. Briefly, cAMP is activated by G-protein coupled receptor ligands which upon activation of cAMP, mediate signal transduction mainly through protein kinase A (PKA). ${ }^{4}$ PKA then activates cAMP response element-binding protein (CREB), a response element that is possessed by numerous genes involved in the pathophysiology of psoriasis, including IL-2, IL-6, IL-10 and TNF $\alpha .{ }^{5}$ Intracellular cAMP concentrations are mediated by adenylyl cyclase (AC) and phosphodiesterases (PDE). PDEs are responsible for degrading cAMP, of which the PDE-4 isoenzyme is the most prominent in immune cells, such as lymphocytes, granulocytes and monocytes/macrophages. ${ }^{6,7}$ PDE-4 inhibitors can therefore suppress inflammatory cytokine generation and secretion, including IFN- $\gamma$, TNF- $\alpha$, and IL-2, IL-12 and IL-23, ${ }^{8}$ as well as reduce superoxide generation and chemotaxis. ${ }^{6,8}$ As well, PDE-4 inhibitors upregulate anti-inflammatory cytokines such as IL-10 through activation of CREB. See Figure 1 for a summary of the PDE-4/ PKA signaling pathway in a pro-inflammatory state and with inhibition of PDE-4.

Both non-selective and selective PDE inhibitors are available on the market for various indications. The nonselective PDE inhibitors include pentoxifylline and theophylline and have no known benefit for psoriasis. Pentoxifylline, used in peripheral vascular disease, is a competitive non-selective PDE inhibitor. ${ }^{9}$ Theophylline is a mostly non-selective PDE inhibitor that has some selective inhibition of PDE1-5 and is used to improve airflow obstruction in asthma, chronic bronchitis and chronic obstructive pulmonary disease (COPD). ${ }^{10}$

Selective PDE-4 inhibitors have been investigated across a wide variety of dermatologic inflammatory conditions in addition to psoriasis, including cutaneous sarcoidosis, discoid lupus erythematosus, scleroderma, Behcet's disease and atopic dermatitis. ${ }^{2,11}$ The main issue of systemic PDE inhibition is gastrointestinal adverse effects, and newer generation, more selective agents have improved tolerability. Multiple clinical trials have supported the use of the PDE-4 inhibitor, apremilast, which has been approved for use in psoriasis, psoriatic arthritis and Behcet's disease. Adverse events including nausea, vomiting, headache, weight loss and depression have been reported and may limit use in some patients. Since PDE-4 inhibitors are small molecules, they are amenable to topical application and therefore, topical PDE-4 inhibitors are currently being investigated with the goal to avoid systemic adverse effects. Topical roflumilast is being investigated in psoriasis, atopic dermatitis and seborrheic dermatitis, and topical crisaborole has already received approval for use in atopic dermatitis. Though previous reviews have examined the role of PDE-4 inhibitors in the treatment of psoriasis, this review comprehensively summarizes the currently available data. ${ }^{2,12,13}$ Herein, we summarize the literature exploring the efficacy and safety of PDE-4 inhibition in the treatment of psoriasis, with a specific focus on data from randomized clinical trials.

\section{Apremilast}

Apremilast $\left(\right.$ Otezla $\left.^{\circledR}\right)$ was the first orally available PDE-4 inhibitor approved by the Food and Drug Administration (FDA) in 2014 for the treatment of moderate-to-severe plaque psoriasis. ${ }^{14}$ Through its intracellular mechanism of action, apremilast inhibits the degradation of cAMP, thus increasing the concentration of cAMP and ultimately reducing the expression of pro-inflammatory mediators and increasing the level of anti-inflammatory mediators. ${ }^{13,15-17}$

Two Phase 3, multicentered, randomized, doubleblinded, placebo-controlled trials, ESTEEM 1 and ESTEEM 2, evaluated the efficacy and safety of apremilast $30 \mathrm{mg}$ twice a day (BID) in patients with moderate-to-severe plaque psoriasis. Eligible subjects had psoriasis with a Psoriasis Area and Severity Index (PASI) $\geq 12$, body surface area (BSA) $\geq 10 \%$ and Physician Global Assessment (PGA) $\geq 3$. In 2015, Papp et al reported the ESTEEM 1 trial, which enrolled 844 adults. ${ }^{14}$ During the first 16-week period, the primary endpoint was met with a significantly greater proportion of patients receiving apremilast (33.1\%) achieving a treatment reduction of over $75 \%$ in the PASI score (PASI-75) relative to placebo $(5.3 \%$, $\mathrm{p}<0.0001)$. In the second phase of the study, patients that were initially randomized to placebo were switched to apremilast $30 \mathrm{mg}$ BID for 16 weeks. Subjects switched from placebo to apremilast had improved response rates for PASI75, mean change in PASI score, and improvement in the pruritus visual analogue scale (VAS) score at week 32 . In the final 20-week phase of the study, patients initially randomized to apremilast were re-randomized to receive either apremilast or placebo. Between these cohorts, a significantly greater proportion of patients re-randomized to apremilast $(61.0 \%)$ achieved PASI-75 relative to $11.7 \%$ of patients rerandomized to placebo. A total of $55.7 \%$ of patients initially randomized to placebo and $69.3 \%$ randomized to apremilast had at least one adverse event (AE), most of which were mild or moderate in severity. The most common reported 


\section{A Proinflammatory state}

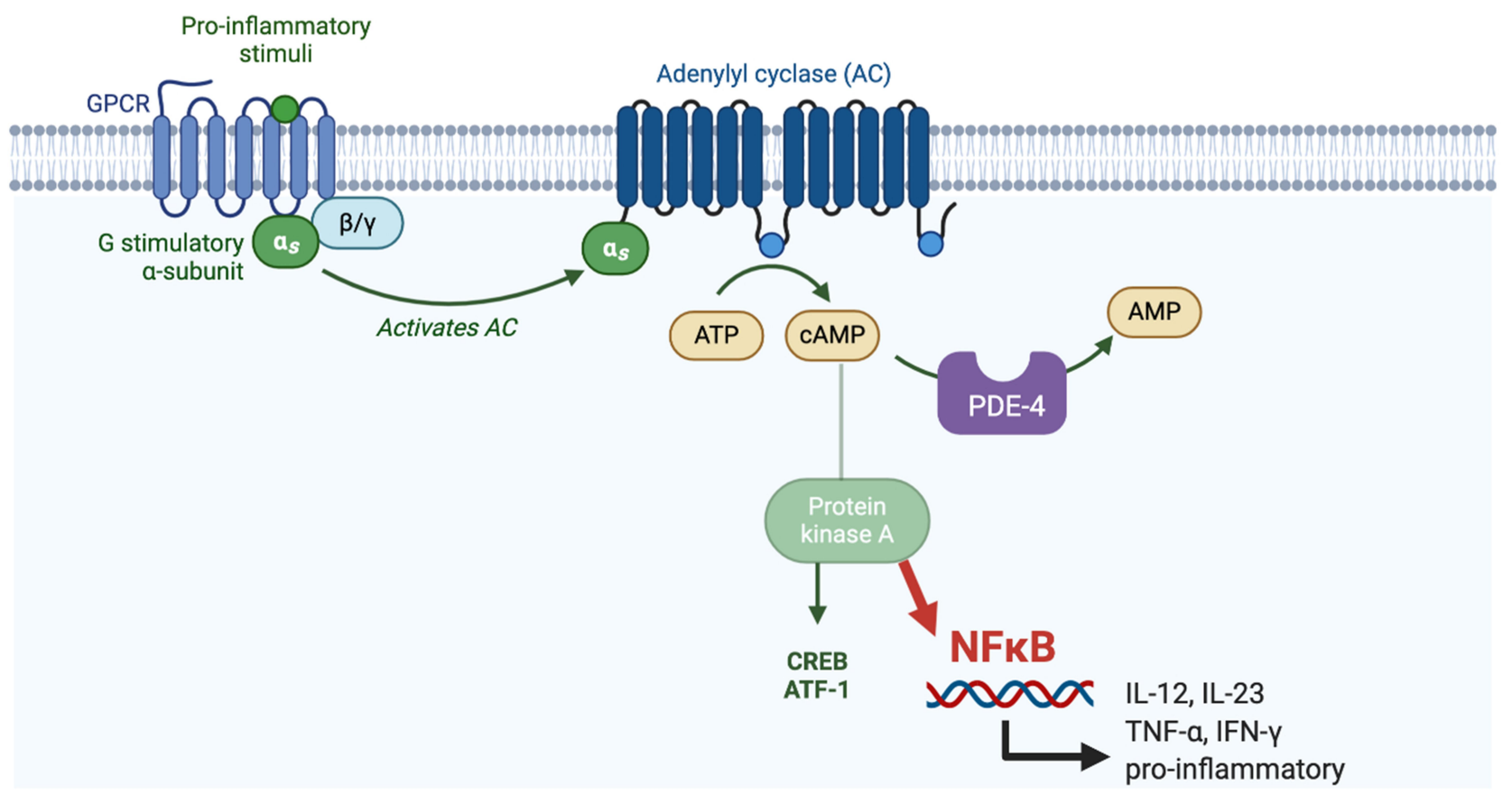

\section{B Inhibition of PDE4}

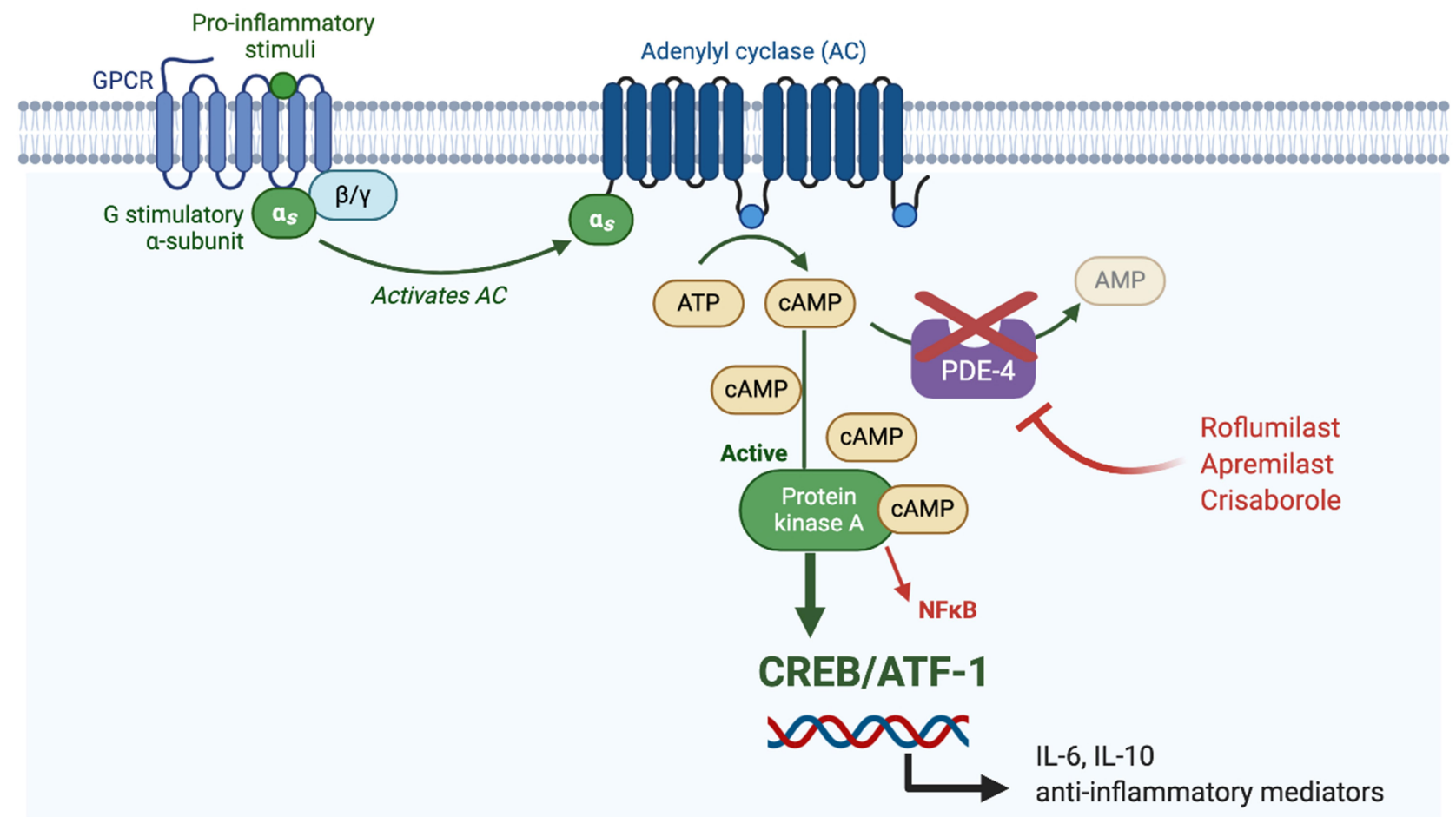

Figure I Overview of the PDE4/PKA cascade pathway. Panel (A). In a proinflammatory state, PDE-4 promotes production of inflammatory cytokines through activation of NFKB and reduces production of anti-inflammatory cytokines such as IL-I0 by degrading cAMP which is responsible for maintaining immune homeostasis. Panel (B). Inhibition of PDE4 leads to elevation of cAMP which triggers the PKA pathway. PKA suppresses the production of proinflammatory mediators and promotes the production of anti-inflammatory mediators, such as IL-10, through gene transcription driven by CREB and ATF-I.

Notes: Adapted from “Activation of Protein Kinase A (PKA)", by BioRender.com (2020). Retrieved from https://app.biorender.com/biorender-templates.

Abbreviations: ATF-I, cAMP-dependent transcription factor I; cAMP, cyclic adenosine monophosphate; CREB, cAMP response element binding protein; IL, interleukin; PDE-4, phosphodiesterase-4; PKA, protein kinase A; NFKB, nuclear factor kappa $B$. 
AEs with apremilast treatment were diarrhea, upper respiratory tract infection (URTI), nausea, nasopharyngitis and headache. The AEs of diarrhea, nausea and headache were more frequent during the first 2 weeks and reduced in frequency over time. The median weight loss in the long-term apremilast-exposure group was $1.40 \mathrm{~kg}$. Nineteen percent of these patients had weight loss greater than $5 \%$. No patient discontinued treatment as a result of the weight loss.

The second pivotal trial, ESTEEM 2, was reported by Paul et al. ${ }^{18}$ Similar results in ESTEEM 1 were reported with the primary endpoint of PASI-75 at week 16 being met with a significantly greater proportion of patients receiving apremilast $(28.8 \%)$ compared to placebo $(5.8 \%, \mathrm{p}<0.0001)$ (See Figure 2). Other significant improvements, such as assessment of Dermatology Life Quality Index (DLQI) showed a clinically meaningful improvement (DLQI improvement $>5$ points) in those subjects with DLQI $>5$ at baseline in $70.8 \%$ of patients $(\mathrm{P}<0.001)$ showing improved quality of life. Efficacy results were generally maintained over the 52-week period in those subjects that were initially PASI 50 responders. AEs reported were similar to those in ESTEEM 1. Weight loss of greater than 5\% was reported in $20.2 \%$ of patients in the long-term apremilast group. Two patients in the apremilast group discontinued treatment due to weight loss.

In 2017 , Reich et al reported on phase $3 \mathrm{~b}$, multicentered, double-blinded, randomized controlled trial (LIBERATE) of 250 adults with moderate-to-severe plaque psoriasis. ${ }^{16}$ For the first 16 weeks, patients were randomized to receive either placebo, apremilast $30 \mathrm{mg}$ BID or etanercept $50 \mathrm{mg}$ weekly (QW). After 16 weeks, a significantly greater proportion of patients achieved PASI-75 with apremilast $(39.8 \%)$ versus placebo $(11.9 \%, \mathrm{p}<0.0001)$ at the primary endpoint (see Figure 2). In the extension phase from week 16 to 104 , all patients were switched to apremilast therapy. Here, it was reported that the PASI-75 response rate was maintained across treatment cohorts throughout week 104 (45.9-51.9\%). Similarly, significant, sustained improvements were maintained in the scalp physician global assessment response (ScPGA), Nail Psoriasis Severity Index (NAPSI), DLQI and pruritus visual analogue scale (VAS) with apremilast treatment. The most common AEs reported were similar to previous studies and included diarrhea, nausea, URTI and bronchitis. Most AEs were mild or moderate in nature, with the distribution of serious adverse events (SAEs) balanced across groups. Relative to baseline, the mean weight loss was $+0.03 \mathrm{~kg}$ for the placebo arm and $-0.78 \mathrm{~kg}$ for the apremilast cohort. In the cohort of patients that received a placebo for the initial 16-week period followed by apremilast therapy, one patient $(1.4 \%)$ reported the development of a psychotic disorder with suicidal ideation.

Rich et al reported a secondary analysis from the ESTEEM trials, specifically the efficacy of apremilast in difficult-to-treat areas of nail and scalp psoriasis in 1255 adults. ${ }^{19}$ In the first 16 weeks, the NAPSI was significantly improved in patients that received apremilast versus placebo (ESTEEM 1: $-22.5 \%$ versus $+6.5 \%$, respectively,

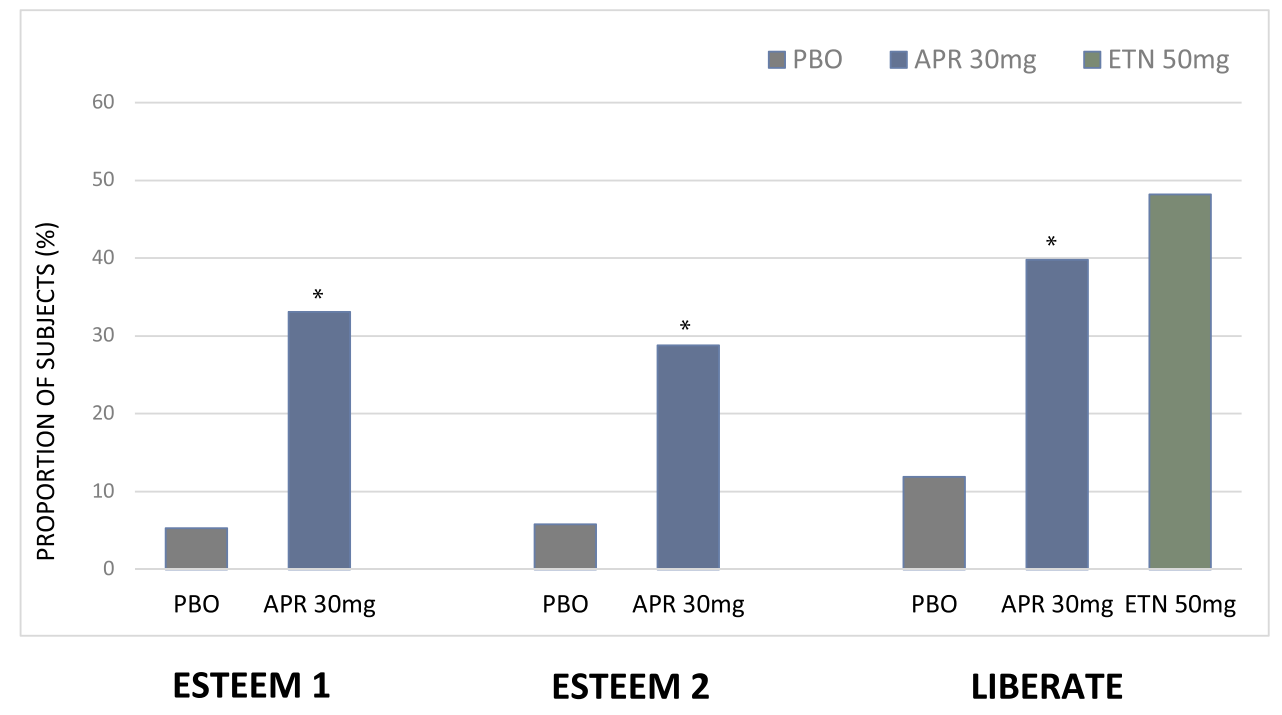

Figure 2 Summary of phase 3 apremilast clinical trials; PASI 75 results at week 16. Notes: $*$ P $<0.000$ I vs placebo. ESTEEM I, ${ }^{14}$ ESTEEM $2,{ }^{18}$ LIBERATE $^{16}$.

Abbreviations: APR, apremilast; ETN, etanercept; PBO, placebo. 
$\mathrm{p}<0.0001$; ESTEEM 2: $-29.0 \%$ versus $-7.1 \%$, respectively, $\mathrm{p}=0.0052$ ). As well, the ScPGA response was significantly greater with apremilast treatment $(p<0.0001)$. Over the course of follow-up, the achievement of $50 \%$ improvement in NAPSI (NAPSI-50) was maintained in patients who were initial PASI responders, irrespective of whether they were initially randomized to apremilast (ESTEEM 1: 70.7\%, ESTEEM 2: 68.6\%) or placebo (ESTEEM 1: 64.1\%, ESTEEM 2: 69.0\%). A similar conclusion was found for patients with scalp psoriasis. ${ }^{19}$

In 2020, Van Voorhees et al reported a phase 3b, double-blinded, placebo-controlled randomized study of 303 patients with moderate-to-severe plaque psoriasis including the scalp. $^{20}$ Following apremilast therapy, a significantly greater proportion of patients achieved the primary endpoint of ScPGA response relative to placebo, defined as a score of either 0 (clear) or 1 (almost clear), $43.3 \%$ versus $13.7 \%$, respectively, $p<0.001$. When considering secondary outcomes, apremilast was associated with a higher proportion of subjects with response on the scalp itch numeric rating scale (NRS) $(47.1 \%$ versus $22.5 \%$, $\mathrm{p}<0.001)$ and whole-body itch NRS $(45.5 \%$ versus $22.5 \%, \mathrm{p}<0.001)$. Quality of life measures also favoured apremilast over placebo (DLQI improvement: -6.7 versus -3.8 , respectively, $\mathrm{p}<0.001)$. Common AEs reported were diarrhea, nausea, headache and vomiting in line with previous trials. In the apremilast group, $2.5 \%$ of patients reported depression. Mean weight loss was $-0.9 \mathrm{~kg}$ versus $-0.2 \mathrm{~kg}$ in the apremilast group vs placebo, respectively.

\section{Roflumilast}

Oral roflumilast (Daxas ${ }^{\circledR}$, Daliresp ${ }^{\circledR}$ ) is a selective PDE-4 inhibitor that was FDA approved as a treatment for COPD exacerbation in 2011. ${ }^{21}$ The effect of oral roflumilast in psoriasis has not previously been reported. However, recently an investigator-initiated study (PSORRO, NCT04549870) comparing oral roflumilast $500 \mathrm{mcg}$ to placebo has been registered with a primary endpoint of PASI-75 at week 12. Results are not yet available. $^{22}$

Topical roflumilast (ARQ-151, Arcutis Biotherapeutics) is a highly potent PDE-4 inhibitor currently under investigation for the treatment of plaque psoriasis. Based on IC50 values of inhibitor affinity for the PDE-4 molecule, roflumilast is approximately $25-300 \mathrm{x}$ more potent than either apremilast or crisaborole depending on the comparator and PDE-4 isoform analyzed. ${ }^{23}$ ARQ-151 is a once-daily cream consisting of roflumilast in a high-water content moisturizing cream base vehicle containing diethylene glycol monoethyl ether or Transcutol ${ }^{\circledR}$ to enhance penetration in the skin. ${ }^{21,24}$ A Phase 1/2a randomized controlled study investigating the efficacy and safety of once-daily roflumilast cream in patients with plaque psoriasis was recently reported. ${ }^{25}$ This study included patients with a history of chronic plaque psoriasis of $>6$-month duration with $\leq 5 \%$ BSA involvement. Cohort $1(\mathrm{n}=8)$ was a single-dose, openlabel cohort that received $0.5 \%$ roflumilast cream to a $25 \mathrm{~cm}^{2}$ psoriatic plaque. Cohort $2(\mathrm{n}=89)$ was a doubleblinded portion that randomized patients to receive either roflumilast cream $0.5 \%, 0.15 \%$ or vehicle for 28 days. The primary endpoint was met with a statistically significant improvement in the Target Plaque Severity Score (TPSS) $\mathrm{x}$ target plaque area (TPA) following administration of roflumilast $0.5 \%(\mathrm{p}=0.0007)$ and $0.15 \%$ cream $(\mathrm{p}=0.0011)$ versus vehicle (Figure 3A). Differences between the two comparators were reached as early as 2 weeks. At both doses, a $66-67 \%$ improvement in TPSS x TPA from baseline was observed at week 4 versus $38 \%$ for vehicle. AEs in cohorts 1 and 2 were all mild or moderate and were similar between active arms and placebo. The most common AEs were application-site erythema, application-site pain, nasopharyngitis and muscle strain. ${ }^{25}$

A Phase 2b, parallel-group, double-blinded, vehiclecontrolled trial was conducted to further investigate the safety and efficacy of once-daily topical roflumilast in plaque psoriasis. ${ }^{26}$ Subjects were randomly assigned to use roflumilast $0.3 \%$ cream, roflumilast $0.15 \%$ cream or vehicle cream once daily for 12 weeks. Eligible subjects were 18 years of age or older and had plaque psoriasis for a minimum of 6 months of at least a mild severity (score $\geq 2$ ) on a 5-point Investigators Global Assessment (IGA) scale $(0=$ clear, $4=$ severe). As well, patients had to have a score of at least 2 on the modified PASI (mPASI). A total of 331 patients were randomized 1:1:1 to three treatment arms: 109 to $0.3 \%$ cream, 113 to $0.15 \%$ cream and 109 to vehicle cream. At 6 weeks, the percentage of patients achieving an IGA score of clear or almost clear (IGA score 0 or 1) was $28 \%$ in the roflumilast $0.3 \%$ group $(\mathrm{P}<0.001), \quad 23 \%$ in the roflumilast $0.15 \%$ group $(\mathrm{P}=0.004)$ compared to $8 \%$ in the vehicle group. For patients with psoriasis in intertriginous areas, the endpoint of an IGA score of clear or almost clear disease plus a 2-grade improvement in the intertriginous area IGA score at week 6 occurred in $73 \%$ of patients in the $0.3 \%$ roflumilast group compared to $44 \%$ in the $0.15 \%$ group and $29 \%$ of those in the vehicle group. The mean change 

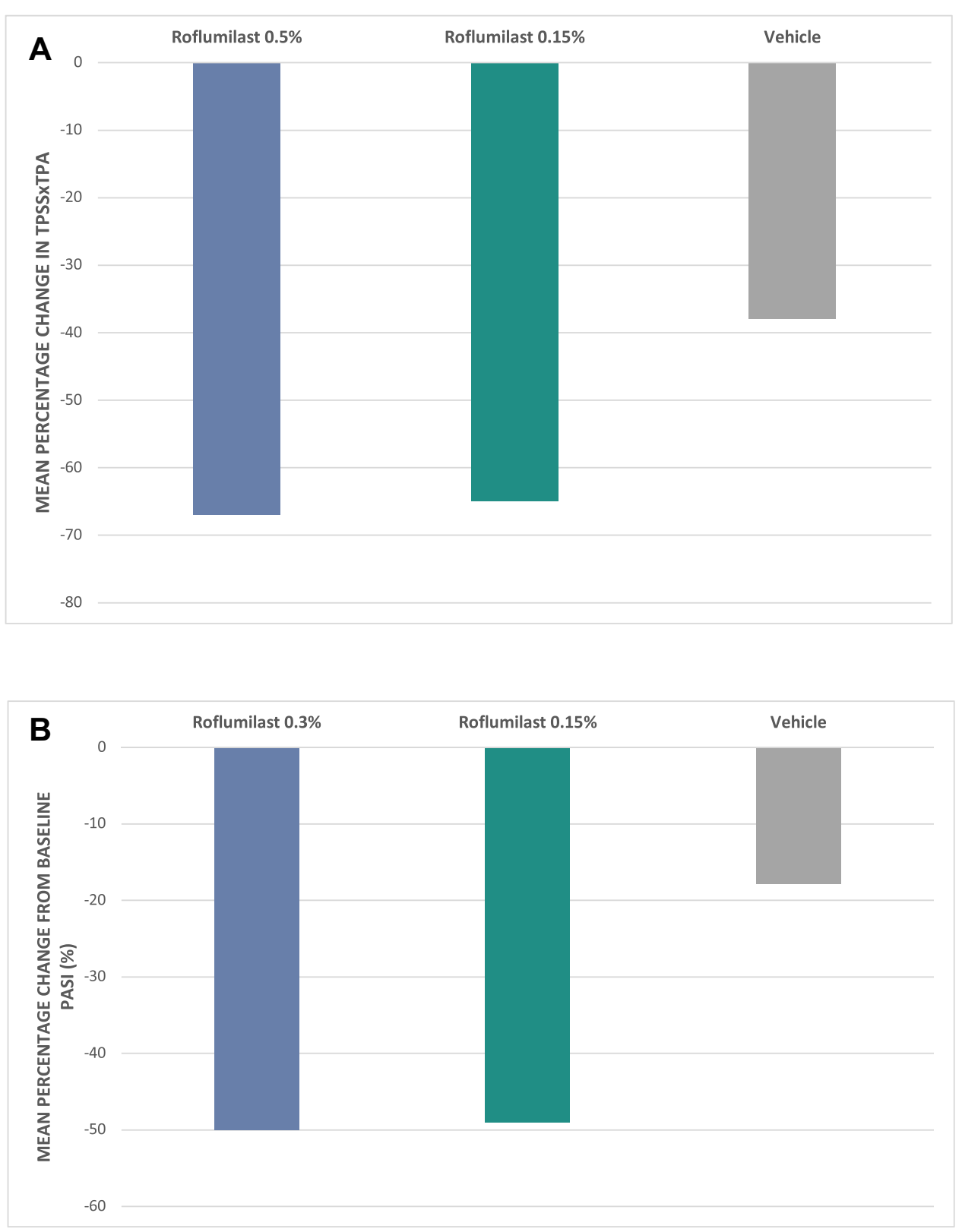

Figure 3 (A) Summary of Roflumilast Clinical Trials - Mean Percentage Change from Baseline in TPSSxTPA at week 4. (B) Summary of Roflumilast Clinical Trials - Mean Percentage Change from Baseline PASI at week 6.

Notes: $(\mathbf{A}){ }^{*} \mathrm{p}<0.0 \mathrm{l}$ versus placebo. Data derived from Papp et al. ${ }^{25}$ (B) Data derived from Lebwohl et al. ${ }^{26}$

Abbreviations: (A) TPSS, target plaque severity score; TPA, target plaque area. (B) *PASI, psoriasis area and severity index.

from baseline PASI score at week 6 was $-50.0 \%$ and $-49.0 \%$ in the $0.3 \%$ and $0.15 \%$ roflumilast group, respectively, compared to the vehicle group $(-17.8 \%$ from baseline, Figure 3B). ${ }^{26}$ In terms of safety, the frequency of AEs was similar across all groups: $39 \%$ in the $0.3 \%$ roflumilast group, $27 \%$ in the $0.15 \%$ group and $20 \%$ in the vehicle group. One SAE occurred in each of the two roflumilast treatment groups and two SAEs occurred in the placebo group. The most common AEs were nasopharyngitis and URTI. Gastrointestinal side effects such as nausea and diarrhea had a less than $1 \%$ incidence in this study, significantly less than is commonly seen with apremilast. ${ }^{26}$

\section{Crisaborole}

Crisaborole ointment 2\% (AN2728, Eucrisa ${ }^{\circledR}$, Pfizer, New York), is a PDE-4 inhibitor that was FDA approved in 2016 for mild-to-moderate atopic dermatitis with an extension of indication to children at least 3 months of age in $2020 .{ }^{27}$ The inhibition of PDE-4 is made possible by the unique configuration of boron within the crisaborole 
molecule. ${ }^{3}$ The boron enables the synthesis of a low molecular weight compound, allowing for effective penetration through skin. Similar to others, this PDE-4 inhibitor leads to increased cAMP-dependent activation of PKA and inhibits downstream NFAT and NF-kB signaling pathways, thereby attenuating skin inflammation. ${ }^{28}$

Two randomized, double-blinded, vehicle-controlled phase 3 clinical trials in patients with atopic dermatitis showed that crisaborole ointment $2 \%$ effectively reduced the signs and symptoms of $\mathrm{AD}$ in both children and adults. ${ }^{29,30}$ Early on in development, crisaborole was also investigated for plaque psoriasis, in a phase $2 b$ randomized, double-blinded, vehicle-controlled, bilateral comparison 12-week study (NCT01029405). ${ }^{31}$ Subjects were randomized as their own controls to receive $0.5 \%$ or $2 \%$ ointment vs vehicle ointment OD or BID in a split-body design. The crisaborole $2 \%$ ointment BID regimen was most effective, well tolerated and had no safety concerns but was never developed further for use in psoriasis, and the results were never published in the literature. ${ }^{31}$ Multiple other unpublished studies have also supported the efficacy of crisaborole in plaque psoriasis. ${ }^{32}$ Psoriatic lesion thickness was significantly reduced relative to vehicle in two phase $1 \mathrm{~b}$ studies ( $\mathrm{n}=24$ total) at crisaborole concentrations ranging from $0.5 \%$ to $5 \%$. In a phase $2 \mathrm{a}$ trial of crisaborole $5 \%(n=35)$, there was a significant reduction in target plaque severity $(68.6 \%$ vs $5.7 \%$, $\mathrm{p}<0.001$ ), which was confirmed in a smaller phase $2 \mathrm{~b}$ trial of 30 patients. ${ }^{32}$ More recently, crisaborole has been investigated for psoriasis in sensitive areas where the application of TCS is often a concern. A double-blinded, randomized, vehicle-controlled study was conducted to investigate the efficacy and safety of $2 \%$ crisaborole ointment in 21 participants with anogenital, intertriginous and facial psoriasis. $^{33}$ After 4 weeks, there was a $66 \%$ improvement in the Target Lesion Severity Scale (TLSS) compared to $8 \%$ in the vehicle group $(\mathrm{P}=0.0011)$. At 8 weeks, there was $81 \%$ lesion improvement with $71 \%$ reaching clinical clearance (TLSS $\leq 1$ ). There were no reports of adverse skin reactions at application sites. ${ }^{33}$ Given that the skin barrier remains intact in psoriasis, the authors hypothesized that the use of crisaborole in psoriasis would result in lower rates of skin irritation than in atopic dermatitis. Topical crisaborole has not received an indication for use in psoriasis but off-label use has been reported. Two case reports in the literature detail the success of crisaborole in facial and intertriginous psoriasis as well as palmoplantar psoriasis when systemic therapy was either not available or discontinued due to adverse effects. $^{34,35}$

\section{Discussion}

Psoriasis is a chronic, relapsing inflammatory skin condition that has a profound impact on the quality of life. The mainstay of treatment in mild disease is currently topical therapy, with systemic treatments used for moderate to severe disease, often with concomitant topical therapy. The use of TCS is limited by long-term cutaneous adverse effects with particular concern in sensitive areas. Surveys show that, despite the variety of systemic agents and biologics available, certain psoriasis patients do not continue with treatment because of poor tolerability, lack of effectiveness or burden of monitoring and treatment administration. ${ }^{36,37}$ As a result of this unmet need, the exploration of alternative treatment agents is continually needed. PDE-4 inhibitors block the degradation of cAMP, leading to a reduction in inflammation. In this review, we aimed to summarize the published literature on PDE-4 inhibitors in psoriasis.

Only apremilast has been approved for use in moderate-to-severe psoriasis as an oral agent and plays an important role in systemic management. Both ESTEEM 1 and ESTEEM 2 demonstrated a higher efficacy response relative to placebo, with differences maintained in difficult-totreat areas, such as scalp and nail psoriasis. ${ }^{14,18}$ With respect to safety, most AEs including diarrhea, nausea and headache, are transient, mild-to-moderate in severity and have a low incidence of treatment discontinuation. ${ }^{14}$ Beyond the efficacy and safety data in psoriasis, apremilast also plays a role in the amelioration of symptoms of psoriatic arthritis. ${ }^{38}$ To further investigate the role of apremilast in the management of mild to moderate psoriasis patients, the ADVANCE trial has been completed; however, results have not yet been published. ${ }^{39}$

Due to the potential AEs associated with oral administration of PDE-4 inhibitors, the topical PDE-4 inhibitor roflumilast has been investigated as an alternative that is associated with a reduced incidence of AEs, including gastrointestinal symptoms. Topical roflumilast was found to have a clinically significant level of efficacy starting at 2 weeks after randomization. ${ }^{25}$ It has been suggested that based on TPSSxTPA scores, roflumilast performs comparably to that of betamethasone dipropionate after 4 weeks of therapy. ${ }^{25,40}$ The favourable safety profile of topical roflumilast is likely derived from its pharmacokinetic advantages. Unlike oral forms, which may be associated with spikes in 
trough-to-peak blood levels following initial administration, topical roflumilast cream has a slow ascent to maximal plasma concentrations over days. ${ }^{25}$

Crisaborole is a topical PDE-4 inhibitor currently approved for atopic dermatitis, which has been previously investigated for use for plaque psoriasis including a study in sensitive areas. A recent pilot study showed the efficacy of crisaborole $2 \%$ was found to be superior relative to vehicle. ${ }^{33}$ Though there have been multiple trials that have shown efficacy in psoriasis, most have been unpublished and it is unlikely that further research is planned. ${ }^{41}$ Considering that the thickness of the stratum corneum is inversely proportional to the absorption of topical agents, topical crisaborole is ideal for thin, non-scaly plaques. ${ }^{42}$ Though studies have reported local burning and stinging following crisaborole administration in atopic dermatitis, no reports of adverse skin reactions were observed in the pilot study of plaque psoriasis patients. ${ }^{33,43}$ This is likely due to the fact that eczematous skin has an inherent barrier dysfunction that is not similarly found in psoriatic skin.

Limitations of this review include a paucity of randomized controlled trials for PDE-4 inhibitors, especially for some agents with only preliminary data, such as crisaborole. Most studies report on a limited duration and a limited number of participants challenging generalizability to the clinic population. It is important to note that, in most cases, studies were limited to plaque psoriasis, and the external validity of these results in non-plaque variants is unknown.

\section{Conclusion}

Overall, oral and topical PDE-4 inhibitors are promising therapies in the treatment armamentarium for plaque psoriasis. With proven efficacy and a promising safety profile, these agents hold promise and warrant further investigation. Though oral apremilast is the only PDE-4 inhibitor currently approved for use in psoriasis, we eagerly await results from larger trials of topical roflumilast in this setting.

\section{Acknowledgments}

No acknowledgements to disclose.

\section{Disclosure}

Dr Melinda J Gooderham has been an investigator, speaker, consultant or advisory board member for AbbVie, Amgen, Akros, Arcutis, Boehringer Ingelheim, BMS, Celgene, Dermira, Dermavant, Galderma, GSK, Eli Lilly, Incyte,
Janssen, Kyowa Kirin, Leo Pharma, Medimmune, Merck, Novartis, Pfizer, Regeneron, Roche, Sanofi Genzyme, Sun Pharma, UCB, and Valeant/Bausch. The authors have no other conflicts of interest to disclose in this work.

\section{References}

1. Lowes MA, Suárez-Fariñas M, Krueger JG. Immunology of psoriasis. Annu Rev Immunol. 2014;32:227-255. doi:10.1146/annurev-immunol $-032713-120225$

2. Wittmann M, Helliwell PS. Phosphodiesterase 4 inhibition in the treatment of psoriasis, psoriatic arthritis and other chronic inflammatory diseases. Dermatol Ther (Heidelb). 2013;3(1):1-15. doi:10.1007/ s13555-013-0023-0

3. Yazdanian N, Mozafarpoor S, Goodarzi A. Phosphodiesterase inhibitors and prostaglandin analogues in dermatology: a comprehensive review. Dermatol Ther. 2021;1:e14669. doi:10.1111/dth.14669

4. Schafer P. Apremilast mechanism of action and application to psoriasis and psoriatic arthritis. Biochem Pharmacol. 2012;83 (12):1583-1590. doi:10.1016/j.bcp.2012.01.001

5. Wen AY, Sakamoto KM, Miller LS. The role of the transcription factor CREB in immune function. $J$ Immunol. 2010;185(11): 6413-6419. doi:10.4049/jimmunol.1001829

6. Baumer W, Hoppmann J, Rundfeldt C, Kietzmann M. Highly selective phosphodiesterase 4 inhibitors for the treatment of allergic skin diseases and psoriasis. Inflamm Allergy Drug Targets. 2007;6(1):17-26.

7. Torphy TJ. Phosphodiesterase isozymes: molecular targets for novel antiasthma agents. Am J Respir Crit Care Med. 1998;157 (2):351-370. doi:10.1164/ajrccm.157.2.9708012

8. Schafer P, Parton A, Gandhi A, et al. Apremilast, a cAMP phosphodiesterase-4 inhibitor, demonstrates anti-inflammatory activity in vitro and in a model of psoriasis. Br J Pharmacol. 2010;159 (4):842-855. doi:10.1111/j.1476-5381.2009.00559.x

9. Magalhães GM, Da Silva Carneiro SC, Do Amaral KP, de Freire Cássia F, Machado-Pinto J, Cuzzi T. Psoriasis and pentoxifylline: a clinical, histopathologic, and immunohistochemical evaluation. Skinmed. 2006;5(6):278-284. doi:10.1111/j.1540-9740.2006.05681. $\mathrm{x}$

10. Barnes PJ. Theophylline. Am J Respir Crit Care Med. 2013;188 (8):901-906. doi:10.1164/rccm.201302-0388PP

11. Hatemi G, Melikoglu M, Tunc R, et al. Apremilast for Behçet's syndrome - a phase 2, placebo-controlled study. $N$ Engl $J$ Med. 2015;372(16):1510-1518. doi:10.1056/NEJMoa1408684

12. Deeks ED. Apremilast: a review in psoriasis and psoriatic arthritis. Drugs. 2015;75(12):1393-1403. doi:10.1007/s40265-015-0439-1

13. Gooderham M, Papp K. Apremilast in the treatment of psoriasis and psoriatic arthritis. Skin Therapy Lett. 2015;20(5):1-6.

14. Papp K, Reich K, Leonardi CL, et al. Apremilast, an oral phosphodiesterase 4 (PDE4) inhibitor, in patients with moderate to severe plaque psoriasis: results of a Phase III, randomized, controlled trial (Efficacy and Safety Trial Evaluating the Effects of Apremilast in Psoriasis [ESTEEM] 1). J Am Acad Dermatol. 2015;73(1):37-49. doi:10.1016/j.jaad.2015.03.049

15. Gooderham M, Papp K. Selective phosphodiesterase inhibitors for psoriasis: focus on apremilast. BioDrugs. 2015;29(5):327-339. doi:10.1007/s40259-015-0144-3

16. Reich K, Gooderham M, Green L, et al. The efficacy and safety of apremilast, etanercept and placebo in patients with moderate-tosevere plaque psoriasis: 52-week results from a phase IIIb, randomized, placebo-controlled trial (LIBERATE). J Eur Acad Dermatol Venereol. 2017;31(3):507-517. doi:10.1111/jdv.14015

17. Pincelli C, Schafer PH, French LE, Augustin M, Krueger JG. Mechanisms underlying the clinical effects of apremilast for psoriasis. J Drugs Dermatol. 2018;17(8):835-840. 
18. Paul C, Cather J, Gooderham M, et al. Efficacy and safety of apremilast, an oral phosphodiesterase 4 inhibitor, in patients with moderate-to-severe plaque psoriasis over 52 weeks: a phase III, randomized controlled trial (ESTEEM 2). Br J Dermatol. 2015;173(6):13 87-1399. doi:10.1111/bjd.14164

19. Rich P, Gooderham M, Bachelez H, et al. Apremilast, an oral phosphodiesterase 4 inhibitor, in patients with difficult-to-treat nail and scalp psoriasis: results of 2 Phase III randomized, controlled trials (ESTEEM 1 and ESTEEM 2). J Am Acad Dermatol. 2016;74 (1):134-142. doi:10.1016/j.jaad.2015.09.001

20. Van Voorhees AS, Stein Gold L, Lebwohl M, et al. Efficacy and safety of apremilast in patients with moderate to severe plaque psoriasis of the scalp: results of a phase $3 \mathrm{~b}$, multicenter, randomized, placebo-controlled, double-blind study. J Am Acad Dermatol. 2020;83 (1):96-103. doi:10.1016/j.jaad.2020.01.072

21. Oba Y, Lone NA. Efficacy and safety of roflumilast in patients with chronic obstructive pulmonary disease: a systematic review and meta-analysis. Ther Adv Respir Dis. 2013;7(1):13-24. doi:10.1177/ 1753465812466167

22. (U.S) NLoM. Efficacy of roflumilast in the treatment of psoriasis (PSORRO). Identifier NCT04549870; 2020. Available from: https:// clinicaltrials.gov/ct2/show/record/NCT04549870. Accessed January 8, 2021.

23. Dong C, Virtucio C, Zemska O, et al. Treatment of skin inflammation with benzoxaborole phosphodiesterase inhibitors: selectivity, cellular activity, and effect on cytokines associated with skin inflammation and skin architecture changes. J Pharmacol Exp Ther. 2016;358 (3):413-422. doi:10.1124/jpet.116.232819

24. Arcutis. Topical roflumilast cream (ARQ-151) archives - Arcutis biotherapeutics; 2021. Available from: https://arcutis.com/trial/arq151/. Accessed January 8, 2021.

25. Papp KA, Gooderham M, Droege M, et al. Roflumilast cream improves signs and symptoms of plaque psoriasis: results from a phase $1 / 2$ a randomized, controlled study. J Drugs Dermatol. 2020;19(8):734-740. doi:10.36849/JDD.2020.5370

26. Lebwohl MG, Papp KA, Stein gold L, et al. Trial of roflumilast cream for chronic plaque psoriasis. $N$ Engl J Med. 2020;383 (3):229-239. doi:10.1056/NEJMoa2000073

27. Fishbein AB, Silverberg JI, Wilson EJ, Ong PY. Update on atopic dermatitis: diagnosis, severity assessment, and treatment selection. $J$ Allergy Clin Immunol Pract. 2020;8(1):91-101. doi:10.1016/j. jaip.2019.06.044

28. Dina Coronado B, Zane LT, Coronado D. Crisaborole topical ointment, 2\%: a nonsteroidal, topical, anti-inflammatory phosphodiesterase 4 inhibitor in clinical development for the treatment of atopic dermatitis. J Drugs Dermatol. 2016;15:390-396.

29. Paller AS, Tom WL, Lebwohl MG, et al. Efficacy and safety of crisaborole ointment, a novel, nonsteroidal phosphodiesterase 4 (PDE4) inhibitor for the topical treatment of atopic dermatitis (AD) in children and adults. J Am Acad Dermatol. 2016;75(3):494-503. e496. doi:10.1016/j.jaad.2016.05.046

30. Stein Gold L, Spelman L, Spellman MC, Hughes MH, Zane LT. A phase 2, randomized, controlled, dose-ranging study evaluating crisaborole topical ointment, $0.5 \%$ and $2 \%$ in adolescents with mild to moderate atopic dermatitis. J Drugs Dermatol. 2015;14(12):1394.
31. Pfizer. Safety and efficacy study of a novel ointment to treat plaque type psoriasis (AN2728PSR203). U.S. National Library of Medicine; 2009. Available from: https://clinicaltrials.gov/ct2/show/results/ NCT01029405?view=results. Accessed January 19, 2021.

32. Rafael A, Torres T. Topical therapy for psoriasis: a promising future. Focus on JAK and phosphodiesterase-4 inhibitors. Eur J Dermatol. 2016;26(1):3-8. doi:10.1684/ejd.2015.2663

33. Hashim PW, Chima M, Kim HJ, et al. Crisaborole $2 \%$ ointment for the treatment of intertriginous, anogenital, and facial psoriasis: a double-blind, randomized, vehicle-controlled trial. $J$ Am Acad Dermatol. 2020;82(2):360-365. doi:10.1016/j.jaad.2019.06.1288

34. Lee EB, Lebwohl MG, Wu JJ. Treatment of psoriasis with crisaborole. J Dermatolog Treat. 2019;30(2):156-157. doi:10.1080/ 09546634.2018.1480747

35. Robbins AB, Gor A, Bui MR. Topical crisaborole - a potential treatment for recalcitrant palmoplantar psoriasis. JAMA Dermatol. 2018;154(9):1096-1097. doi:10.1001/jamadermatol.2018.2397

36. Lebwohl MG, Bachelez H, Barker J, et al. Patient perspectives in the management of psoriasis: results from the population-based Multinational Assessment of Psoriasis and Psoriatic Arthritis Survey. J Am Acad Dermatol. 2014;70(5):871-881. e830. doi:10. 1016/j.jaad.2013.12.018

37. Armstrong AW, Robertson AD, Wu J, Schupp C, Lebwohl MG. Undertreatment, treatment trends, and treatment dissatisfaction among patients with psoriasis and psoriatic arthritis in the United States: findings from the National Psoriasis Foundation surveys, 2003-2011. JAMA Dermatol. 2013;149(10):1180-1185. doi:10.10 01/jamadermatol.2013.5264

38. Kavanaugh A, Mease PJ, Gomez-Reino JJ, et al. Treatment of psoriatic arthritis in a phase 3 randomised, placebo-controlled trial with apremilast, an oral phosphodiesterase 4 inhibitor. Ann Rheum Dis. 2014;73(6):1020-1026. doi:10.1136/annrheumdis-2013-205056

39. Amgen. Amgen announces positive top-line results from Otezla (apremilast) phase 3 ADVANCE study in mild-to-moderate plaque psoriasis. Available from: https://www.amgen.com/newsroom/ press-releases/2020/05/amgen-announces-positive-topline-resultsfrom-otezla-apremilast-phase-3-advance-study-in-mildtomoderateplaque-psoriasis. Accessed January 7, 2021.

40. Anstey AV, Kragballe K. Retrospective assessment of PASI 50 and PASI 75 attainment with a calcipotriol/betamethasone dipropionate ointment. Int J Dermatol. 2006;45(8):970-975. doi:10.1111/j.13654632.2006.02939.x

41. Search of: AN-2728 psoriasis U.S. National Library of Medicine. Available from: https://clinicaltrials.gov/ct $2 /$ results?cond= Psoriasis $\&$ term $=$ AN $-2728 \&$ cntry $=\&$ state $=\&$ city $=\&$ dist $=$. Accessed January 20, 2021.

42. Andrews SN, Jeong E, Prausnitz MR. Transdermal delivery of molecules is limited by full epidermis, not just stratum corneum. Pharm Res. 2013;30(4):1099-1109. doi:10.1007/s11095-012-0946-7

43. Zane LT, Hughes MH, Shakib S. Tolerability of crisaborole ointment for application on sensitive skin areas: a randomized, double-blind, vehicle-controlled study in healthy volunteers. Am J Clin Dermatol. 2016;17(5):519-526. doi:10.1007/s40257-016-0204-6

\section{Publish your work in this journal}

Psoriasis: Targets and Therapy is international, peer-reviewed, open access journal focusing on psoriasis, nail psoriasis, psoriatic arthritis and related conditions, identification of therapeutic targets and the

optimal use of integrated treatment interventions to achieve improved outcomes and quality of life. Visit http://www.dovepress. com/testimonials.php to read real quotes from published authors. 